Source: Allahyari, E., Hanachi P., Ariakia, F., Kashfi, T. E., Ferns, G. A., Bahrami, A., \& Mobarhan, M. G. (2020). The relationship between neuropsychological function and responsiveness to vitamin D supplementation using artificial neural networks. Nutrition and Health, 26(4), 285-294. doi: 10.1177/0260106020937190

\title{
The relationship between neuropsychological function and responsiveness to vitamin $D$ supplementation using artificial neural networks
}

Elahe Allahyari ${ }^{1}$, Parichehr Hanachi ${ }^{2}$, Fatemeh Ariakia ${ }^{3}$, Toktam Emami Kashfi ${ }^{4}$, Gordon A Ferns ${ }^{5}$, Afsane Bahrami $^{6}$, Majid Ghayour Mobarhan ${ }^{7}$

${ }^{1}$ Department of Epidemiology and Biostatistics, School of Health, Social Determinants of Health Research Center, 125609Birjand University of Medical Sciences, Birjand, Iran.

${ }^{2}$ Department of Biotechnology, Faculty of Biological Sciences, Alzahra University, Vanak, Tehran, Iran.

${ }^{3}$ Department of Biochemistry, School of Medical, Iran University of Medical Sciences, Tehran, Iran.

${ }^{4}$ Department of Motor Behavior, Faculty of Sport Sciences, 48440Ferdowsi University of Mashhad, Mashhad, Iran.

${ }^{5}$ 1949Brighton \& Sussex Medical School, Division of Medical Education, Falmer, Brighton, Sussex BN1 9PH, UK.

${ }^{6}$ Cellular and Molecular Research Center, 125609Birjand University of Medical Sciences, Birjand, Iran.

${ }^{7}$ Metabolic Syndrome Research Center, 37552Mashhad University of Medical Sciences, Mashhad, Iran.

\begin{abstract}
Background: Vitamin D has recently attracted interest for its pleiotropic effects. Vitamin D supplements are a potentially important public health intervention, but the response to supplementation varies between individuals. Aim: We aimed to assess the association between several neuropsychological parameters and the magnitude of response to vitamin D supplementation using an artificial neural network method. Methods: Neuropsychological function was assessed in 619 participants using standard questionnaires. The study participants received vitamin D capsules containing 50,000 IU vitamin D per week over 9 weeks. To assess the relationship between responsiveness to vitamin D supplements and the impact on these neuropsychological parameters, the best-performing artificial neural network algorithms were selected from a combination of different transfer functions in hidden and output layers and variable numbers of hidden layers (between two and 50). The performance of the artificial neural network algorithm was assessed by receiver operating characteristic analysis and variables of importance were identified. Results: The artificial neural network algorithm with sigmoid transfer function in both hidden and output layers could predict responsiveness to vitamin D supplementation effectively. The sensitivity and specificity were between 0.60 and 0.70 and 0.66 and 0.70 , respectively. Cognitive abilities (42.5\%), basal vitamin D (21.3\%), body mass index (9.5\%), and daytime sleepiness (8\%) are the most widely used variables to predict changes in serum vitamin D levels. Conclusions: Cognitive abilities status and baseline 25hydroxyvitamin D are important novel modifiers of the enhancement in circulating 25hydroxyvitamin D after vitamin D supplementation.
\end{abstract}

Keywords: Cognitive abilities, depression, sleep disorders, sleepiness, insomnia, artificial intelligence. 